\title{
Decellularized nerve extracellular matrix/chitosan crosslinked by genipin to prepare a moldable nerve repair material
}

\author{
Fangsong Zhang $\cdot$ Naili Zhang $\cdot$ Qing Xu $\cdot$ Luping Zhang $\cdot$ Chunlei Zhang \\ Hongfu Liu $\cdot$ Zhenhai Yu $\cdot$ Shuai Zhou $\cdot$ Guoying Feng $\cdot$ Fei Huang
}

Received: 8 May 2020/ Accepted: 4 December 2020/Published online: 11 June 2021

(C) The Author(s), under exclusive licence to Springer Nature B.V. 2021

\begin{abstract}
Decellularized nerve extracellular matrix (NECM) composited with chitosan are moldable materials suitable for spinal cord repair. But the rapid biodegradation of the materials may interrupt neural tissue reconstruction in vivo. To improve the stability of the materials, the materials produced by NECM and chitosan hydrogels were crosslinked by genipine, glutaraldehyde or ultraviolet ray. Physicochemical property, degradation and biocompatibility of materials crosslinked by genipin, glutaraldehyde or ultraviolet ray were evaluated. The scaffold crosslinked by genipin possessed a porous structure, and the porosity ratio was $89.07+4.90 \%$, the average
\end{abstract}

Fangsong Zhang, Naili Zhang, Qing Xu, and Luping Zhang have contributed to this work equally and should be considered as co-first authors.

F. Zhang $\cdot$ N. Zhang $\cdot$ L. Zhang $\cdot$ C. Zhang $\cdot$

H. Liu $\cdot$ Z. Yu $\cdot$ S. Zhou $\cdot$ G. Feng $\cdot$ F. Huang $(\bowtie)$

Department of Human Anatomy, College of Basic

Medical Sciences, Binzhou Medical University,

Yantai 264003, People's Republic of China

e-mail: hfei22518@163.com

Q. Xu

Yantai Affiliated Hosptial of Binzhou Medical University, Yantai 264100, People's Republic of China

F. Zhang

Department of Medical Imagine, Shanghai Mental Health Center, School of Medicine, Shanghai Jiao Tong

University, Shanghai 200030, People's Republic of China diameter of pore was $85.32+5.34 \mu \mathrm{m}$. The crosslinked degree of the scaffold crosslinked by genipin and glutaraldehyde was $75.13 \pm 4.87 \%, 71.25 \pm$ $5.06 \%$ respectively; Uncrosslinked scaffold disintegrated when immerged in distilled water while the scaffold crosslinked by genipin and glutaraldehyde group retained their integrity. The scaffold crosslinked by genipin has better water absorption, water retention and anti-enzymatic hydrolysis ability than the other three groups. Cell cytotoxicity showed that the cytotoxicity of scaffold crosslinked by genipin was lower than that crosslinked by glutaraldehyde. The histocompatibility of scaffold crosslinked by genipin was also better than glutaraldehyde group. More cells grew well in the scaffold crosslinked by genipin when co-cultured with L929 cells. The decellularized nerve extracellular matrix/chitosan scaffold crosslinked by

N. Zhang - L. Zhang - C. Zhang · H. Liu ·

Z. Yu $\cdot$ S. Zhou $\cdot$ G. Feng $\cdot$ F. Huang Institute of Human Anatomy and Histology and Embryology, Binzhou Medical University, Yantai 264003, People's Republic of China 
the genipin has good mechanical properties, micro structure and biocompatibility, which is an ideal scaffold for the spinal cord tissue engineering.

Keywords Decellularized nerve extracellular matrix $\cdot$ Chitosan $\cdot$ Cross-linking $\cdot$ Genipin

\section{Introduction}

Repair of spinal cord injury (SCI) in adult mammals is still challenging due to extensive cell loss, axonal disruption, glial scar formation, and deficiency of the regeneration in the mature neurons (Barclay et al. 2016; Cripps et al. 2011). The defect or cystic cavity that develops following injuries to spinal cord is also a major obstacle for tissue repair (Kashif et al. 2019; Milbreta et al. 2014). More than $50 \%$ of patients develop posttraumatic spinal cord cysts or syringomyelia (Badenhorst et al. 2019). Then, restoration of the neural tissue architecture and functions plays a crucial role in the treatment of the SCI.

Various tissue-engineered scaffolds were developed and implanted to reconstruct the damaged neural tissue. One of these scaffolds is decellularized nerve extracellular matrix (NECM), which is derived from the natural nerve, mainly composed of collagen, laminin, fibronectin, chondroitin sulfate and so on. It possesses physicochemical properties similar to autologous nerve with minimal immunological rejection (Lin et al. 2018; Starnecker et al. 2018; Leiva et al. 2018; Zhang et al. 2014). In our recent study, we optimized the decellularization technique of NECM, and confirmed both the NECM and cells-loaded NECM can promote axonal regeneration and construction of spinal cord neuron conduction function in animal models of SCI (Tian et al. 2017; Wang et al. 2014). But NECMs may not fit the irregular and unpredictable geometry cystic cavities of SCI in clinical practice for their poor biological plasticity and the biomechanical properties (Hong et al. 2017; Zhang et al. 2014).

Chitosan (CS) is a polysaccharide obtained from $\mathrm{N}$-deacetylation of chitin, which has been extensive used in treatment of SCI for its good and unique biocompatibility, biodegradability and bioactivity of supporting the adhesion, migration and proliferation of Schwann cells and suppressing the formation of scabs (Fang et al. 2019; Meyer et al. 2016; Schnell et al. 2007). It is a feasible way to prepare a moldable or injectable material by blending CS and hydrogel of NECM for construction the lesion of SCI.

When implanted in the lesion of SCI, the degrading biomaterials should be gradually replaced by endogenous cells to build a new tissue equivalent as opposed to scar (Saldin et al. 2017). Then the control degradation of the scaffold is essential in for the regeneration process. As shown in previous studies, CS and hydrogel of NECM with a poor mechanical property and degrade rapidly in vivo (Tian et al. 2017; Tukmachev et al. 2016). Therefore, a challenging problem that implementation of non-toxoc, biocompatible techniques to prolong the biodegradation of the scaffold appeared.

Crosslinking can increase the stability of biomaterials and improve their mechanical properties. Both chemical and physical crosslinking methods have been used to crosslink CS-based materials (Lau et al. 2018; Li et al. 2015).

Physical crosslinking methods include microwave energy, UV-irradiation, and dehydrothermal (DHT) treatment (Fang et al. 2019). Physical methods do not cause potential harm, whereas their main drawback is the difficulty to obtain the desired crosslinking degree. Aldehydes (formaldehyde, glutaraldehyde, glyceraldehyde), carbodiimides and polyepoxy compound are chemical crosslinkers commonly used. Chemical crosslinking can obtain a high crosslining degree, but the presence of some unreacted crosslinker inside the materials and the risk of formation of toxic products by reaction between the substrate and the crosslinker during biodegradation limited the use of chemical chemical crosslinking (Fajardo et al. 2019; Gao et al. 2017).

For those reasons, naturally derived crosslinking agents draw much interest recently for their low toxicity. Genipin (GP) is a natural crosslinker obtained from geniposide, an iridoid glucoside isolated from the fruits of Genipa Americana and Gardenia jasminoides Ellis, which have been applied as herbal medicine. (Lau et al. 2018; Li et al. 2015). Its ability to crosslink CS and proteins containing residues with primary amine groups has been found recently. And genipin has been recently used as a crosslinker of CS particles for drug delivery and of gelatin for peripheral nerve regeneration (Chiono et al. 2008). Studies have compared genipin with 
other widely used crosslinker such as glutaraldehyde, formaldehyde and epoxy compounds, which proved lower cytotoxity, higher biocompatibility, comparable mechanical strength and resistance against enzymatic degradation of the materials crosslinked by genipin (Lau et al. 2018; Lewandowska-Lancucka et al. 2019; Li et al. 2015). GP crosslinking of extracellular matrix hydrogel was also found had the potential as a scaffold for neural tissue regeneration (Vyborny et al. 2019). However, the study about crosslinking CS/NECM blends was not found.

In this study, we produced the moldable scaffold by CS and NECM, crosslinked by genipin, glutaraldehyde and UV-irradiation to improve the stability. Surface morphology, porosity, swelling, crosslinking degree, dissolution, cytotoxicity, cytocompatibility and histocompatibility of scaffold crosslinked by different methods were compared, with the aim to find out the optimal crosslinking mathod for CS/ NECM blends.

\section{Materials and methods}

Prepare of nerve acellular matrix

Fresh sciatic nerves were harvested from adult pig aged 12-15monthes, slaughtered by China certified butcher (Fuzu Ltd, Yantai, China). The nerves were stored and processed by a modified methods previously reported. In brief, the nerves were immersed in distilled water, shocked for $1 \mathrm{~h}$, the water was substituted with $0.5 \%$ Triton X-100 shocked for another $24 \mathrm{~h}, 4 \%$ sodium deoxycholate $6 \mathrm{~h}$ on the shaker at room temperature. Then the nerves were submerged in liquid nitrogen for $2 \mathrm{~min}$ and transferred to a $37^{\circ} \mathrm{C}$ water bath for $30 \mathrm{~min}$. This freeze-thaw cycle was repeated 5 times. Finally the nerves were digested in DNase-I, RNase-A (Aspergillus niger, Sigma-Aldrich, USA) at $37{ }^{\circ} \mathrm{C}$ for $8 \mathrm{~h}$, rinsed by distilled water 3 times, crushed in the pulverizer, centrifuged, lyophilized by the vacuum freeze dryer, sterilized with $20 \mathrm{kGy}$ irradiation from Cobalt 60 according to sterilization process validation of Osteorad Ltd, and stored at $-20{ }^{\circ} \mathrm{C}$ for 2 weeks until required.

Prepare of extracellular matrix/chitosan scaffolds

The extracellular matrix and chitosan were mixed with the mass ratio $7: 3$, dissolved in $1 \%$ of glacial acetic acid, stirred rapidly $1 \mathrm{~h}$ at room temperature. Genipine (GP) was added to the extracellular matrix/chitosan solution at the concentration of $0.5 \%(\mathrm{w} / \mathrm{v})$, then stirred by the maglev agitator $20 \mathrm{~min}$ until fully mixed, crosslinked at $4{ }^{\circ} \mathrm{C}$ for $24 \mathrm{~h}$. For glutaraldehyde (GTA) crosslinking group, GTA solution was added to the extracellular matrix/chitosan solution at the concentration of $0.5 \%(\mathrm{w} / \mathrm{v})$, then stirred by the maglev agitator 20 min until fully mixed, crosslinked at $4{ }^{\circ} \mathrm{C}$ for $24 \mathrm{~h}$; For ultraviolet crossliking group, the extracellular matrix/chitosan solution was irradiated with the ultraviolet (UV) radiation generator for $6 \mathrm{~h}$ at a dose of 500uw at room temperature. The different groups of extracellular matrix chitosan solution were added into the 6 hole plate, $2 \mathrm{ml}$ each hole, placed at room temperature $12 \mathrm{~h}$ to remove the bubbles, neutralised with a $0.1 \mathrm{M} \mathrm{NaOH}$ (Merck) solution in demineralised water, rinsed several times in demineralized water until $\mathrm{pH}$ was neutral and then dried in a vented oven for $48 \mathrm{~h}$ at $37{ }^{\circ} \mathrm{C}$, then lyophilized by the vacuum freeze dryer.

Scanning electron microscopy (SEM) observation

Each bracket was cut into a sample of $0.2 \times 0.2 \times 0.2 \mathrm{~cm}^{3}$ size. Then surface morphology of the samples was studied using scanning electron microscope (SIGMA 300,CARL ZEISS, Germany) at an accelerating voltage of $10 \mathrm{kV}$ and a vacuum of $60 \mathrm{~Pa}$ at different magnifications. All specimens were pre-coated with a conductive layer of sputtered gold. All experiments were repeated three times.

Swelling tests

The samples $\left(0.5 \times 0.5 \times 0.5 \mathrm{~cm}^{3}\right)$ were weighed and then put in phosphate buffered saline (PBS) at $\mathrm{pH}$ 7.4 at $37^{\circ} \mathrm{C}$. Samples were drawn after $24 \mathrm{~h}$, dried superficially by gentle contact with a filter paper and weighed agian. The swelling percentage was calculated as:

$\mathrm{W}_{1}$, and the water uptake ratio of the specimen was calculated as

$\% \mathrm{~S}_{\mathrm{w}}=\left(\mathrm{W}_{1}-\mathrm{W}_{0}\right) / \mathrm{W}_{0} \times 100 \%$

where $\mathrm{W}_{0}$ and $\mathrm{W}_{1}$ are the sample weights before and after swelling respectively. Each test consisted of three replicate measurements and result was expressed as an average value. 
Porosity tests

The samples $\left(0.5 \times 0.5 \times 0.5 \mathrm{~cm}^{3}\right)$ were weighed and then immersed in anhydrous ethanol. Samples were drawn after reached saturation, dried superficially by gentle contact with a filter paper and weighed agian. The porosity of samples were calculated as:

$\% \mathrm{P}=\left(\mathrm{W}_{1}-\mathrm{W}_{0}\right) / \mathrm{\rho} / \mathrm{V}_{0} \times 100 \%$

where $\mathrm{W}_{0}$ and $\mathrm{W}_{1}$ are the sample weights before and after swelling respectively, $\rho$ is the density of anhydrous ethanol, $V_{0}$ is the volume of the sample. Each test consisted of three replicate measurements and result was expressed as an average value.

\section{Crosslinking degree tests}

$20 \mathrm{mg}$ of samples were weighed and incubated in boiling water for $20 \mathrm{~min}$ with $1 \mathrm{~mL}$ of ninhydrin solution $(1 \mathrm{mg} / \mathrm{mL})$. Then the optical density (OD) at $570 \mathrm{~nm}$ was determined using a spectrophotometer (Bio-Rad Model 550), and the free amino acid content in the specimen was determined according to a predetermined glycine standard curve with known concentrations $(0,0.2,0.4,0.6,0.8,1.0 \mathrm{~mL})$. The crosslinking degree was eventually calculated as: (Chen et al. 2009; Liu and Kim 2012)

$\% \mathrm{Ci}=\left(\mathrm{M}_{0}-\mathrm{M}_{1}\right) / \mathrm{M}_{0} \times 100 \%$

where $\% \mathrm{Ci}$ is the crosslinking index $(\%), \mathrm{M}_{0}$ is free amino acid content in uncrosslinked scaffold materials, $M_{1}$ is free amino acid content in crosslinked scaffold. Each test consisted of three replicate measurements and result was expressed as an average value.

\section{Dissolution tests}

The samples $\left(0.5 \times 0.5 \times 0.5 \mathrm{~cm}^{3}\right)$ were weighed and then incubated in $5 \mathrm{ml}$ mixed solution of $0.2 \mathrm{mg} /$ $\mathrm{ml}$ Collagenase (Solarbio, China) and $1 \mathrm{mg} / \mathrm{ml}$ lysozyme (Solarbio, China). Samples were drawn and lyophilized at different time points $(12 \mathrm{~h}, 1 \mathrm{~d}, 3 \mathrm{~d}$, $5 \mathrm{~d}, 7 \mathrm{~d}, 14 \mathrm{~d})$.The degradation rate of samples at each time point were calculated as (Zhou et al. 2010):

$\mathrm{D} \%=\left(\mathrm{W}_{0}-\mathrm{W}_{1}\right) / \mathrm{W}_{0} \times 100 \%$ where $\mathrm{W}_{0}$ and $\mathrm{W}_{1}$ are the sample weights before and after dissolution at each time point respectively. Each test consisted of three replicate measurements and result was expressed as an average value.

Cytotoxicity and cytocompatibility tests

The extract liquids were prepared by incubating the samples in the culture medium at a ratio of $0.1 \mathrm{~g} / \mathrm{ml}$ $72 \mathrm{~h}$ at $37^{\circ} \mathrm{C}$. $10 \%$ fetal bovine serum (FBS, Hyclone), $100 \mathrm{IU} / \mathrm{ml}$ penicillin and $100 \mathrm{IU} / \mathrm{ml}$ streptomycin were supplemented in the extract liquids. The negative control group was composed of nontoxic polyethylene disks. The reagent control group was composed of the medium.

L929 cells (CC-Y2049, EK-bioscience) were seeded on a $96-$ well culture plate with $100 \mu \mathrm{L}$ with a cell density of $1 \times 10^{4}$ cell $/ \mathrm{mL}$. Extract liquids were changed after incubated in a humidified atmosphere of $5 \% \mathrm{CO}_{2}$ at $37{ }^{\circ} \mathrm{C}$ for $24 \mathrm{~h}$. The cell viability was determined using CCK-8 (Dojindo, Kumamoto, Japan) assay at 1, 3 and 7 days after treatment. At the end of the culture period, $10 \mu \mathrm{L}$ of the CCK-8 solution was added to each well of the culture plate. After $6 \mathrm{~h}$ incubation, absorbance at $450 \mathrm{~nm}$ was measured with a microplate reader (Bio-Rad, Hercules, USA). The cell viability was indicated as a value of absorbance (value of absorbance $=$ absorption of sample-absorption of background). For each group extract, the experiments were repeated three times with five samples at each time point. Culture medium was changed twice a week. Then the cytotoxicity was graded by the International Standard (ISO 10993-5:2009).

The samples were placed singly in a 6-well plate. Then $1 \mathrm{~mL}$ L929 cells suspension with densities about $5 \times 10^{5}$ cells $/ \mathrm{mL}$ were seeded on the samples and $2 \mathrm{~mL}$ culture medium were added to each well $2 \mathrm{~h}$ later. After incubated for $24 \mathrm{~h}$, culture medium was removed the samples with attached cells were rinsed with PBS gently. Attached cells were fixed in $4 \%$ (v/v) paraformaldehyde solution in PBS for $10 \mathrm{~min}$, rinsed with PBS, stained with $0.1 \mathrm{ug} / \mathrm{ml}$ of DAPI (Beyotime $\mathrm{C} 1005)$ for $10 \mathrm{~min}$, rinsed with PBS and then visualized by fluorescence microscopy (ZEISS, LSM 510 META). 
Histocompatibility tests

The samples were implanted into muscles on both sides of the rat spine to evaluate histocompatibility. All procedures involving animals performed in this study were in accordance with NIH guidelines (NIH. Pub. No. 85-23, revised 1996) and approved by the Medical Ethics committee of Binzhou Medical University. 12 SD (Sprague-Dawley) rats, with weighing of $180 \sim 200 \mathrm{~g}$ (Peng Yue experimental animal breeding Co., Ltd, Jinan, China) anesthetized by intraperitoneal injection with $4 \%$ chloral hydrate $(1 \mathrm{~mL}$ per $100 \mathrm{~g})$ and the skin of the back was cut open. Then the samples $\left(0.5 \times 0.5 \times 0.5 \mathrm{~cm}^{3}\right)$ were implanted singly into muscles on both sides of the rat spine. All the operations were handled by the same operator in a sterile environment.

At 1 and 4 weeks after the operation, 6 SD rats were sacrificed, transcardially perfused with $4 \%$ paraformaldehyde (PFA, Sigma) in $0.1 \mathrm{M}$ phosphate buffer. Then tissue around the samples was harvested, fixed in $10 \%$ formalin, embedded in paraffin. And $4 \mu \mathrm{m}$ histological sections were obtained and stained with hematoxylin and eosin (H\&E) according to standard protocols. Three discontinuous sections of each sample were studied using a microscope (DP70, Olympus, Japan) and the Image Capture System (CX41-70, Olympus, Japan). All the slides were examined in a blinded manner.

Statistical analysis

Statistical study was performed using SPSS 18.0 statistical software (Statistical Package for Social Sciences; release 18.0). All data is reported as mean \pm standard deviation of the mean. Significant differences between groups were assessed by a OneWay ANOVA (LSD, least significance difference). Differences were considered significant at $p<0.05$.

\section{Results}

Observed grossly, samples crosslinked by three methods looks smooth, homogeneous and porous. Color of the samples crosslinked by GP was blue (Fig. 1a), color of the samples crosslinked by GTA was dark yellow (Fig. 1b), while color of the samples crosslinked by UV was pale yellow (Fig. 1c). The samples crosslinked by the GP and the UV were soft with good elasticity, and the elasticity of the GTA group was poor.

\section{SEM observation}

Observed by SEM, the microstructure of the sample crosslinked by GP showed a uniform honeycomb porous structure, pore size was about $85.32+5.34 \mu \mathrm{m}$ (Fig. 2a); the sample crosslinked by the GTA (Fig. 2b) and the UV (Fig. 2c) and the sample not crosslinked (Fig. 2d) were arranged plate shaped, did not form the honeycomb porous structure. The thickness layer of the sample crosslinked by the GTA was similar with the sample crosslinked by UV and the sample not crosslinked.

Swelling tests

The swelling tests showed that the swelling degrees of the sample crosslinked by the GP and the GA were $741+85.04 \%$ and $696.75+50.4 \%$ respectively. And the swelling degrees of the sample crosslinked by the UV and the sample not crosslinked were $578.50+50.78 \%$ and $565.40+49.36 \%$ respectively. The swelling degrees of the sample crosslinked by the GP and the GTA were higher than the other two groups. The differances between them were statistically significant $(p<0.05)$. The differances between swelling degrees of the sample crosslinked by the GP and the GTA were not statistically significant ( $p$ $\geq 0.05$ ). And the differances between swelling degrees of the sample crosslinked by the UV and the sample not crosslinked were not statistically significant ( $p \geq 0.05$ ), too (Fig. 3a).

\section{Porosity tests}

The porosity tests showed the porosity rate of the samples crosslinked by GP was highest. In more detail, the porosity rate of the samples crosslinked by GP was $89.07+4.90 \%$, and the samples crosslinked by GTA group was $73.91+6.14 \%$, the samples crosslinked by UV was $80.71 \%+7.27 \%$, and the sample not crosslinked was $74.48+5.63 \%$. The porosity rate of the samples crosslinked by GP was higher than the other three groups. The differances were statistically significant $(p<0.05)$ (Fig. 3b). 

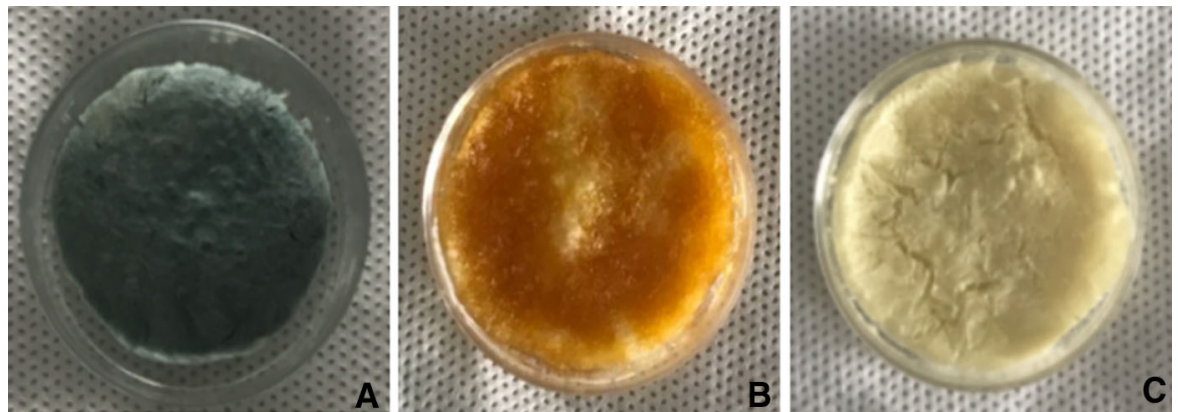

Fig. 1 The cross-linked DNEM/chitosan scaffolds. a Scaffold cross-linked by GP; b scaffold cross-linked by GTA; c Scaffold crosslinked by UV
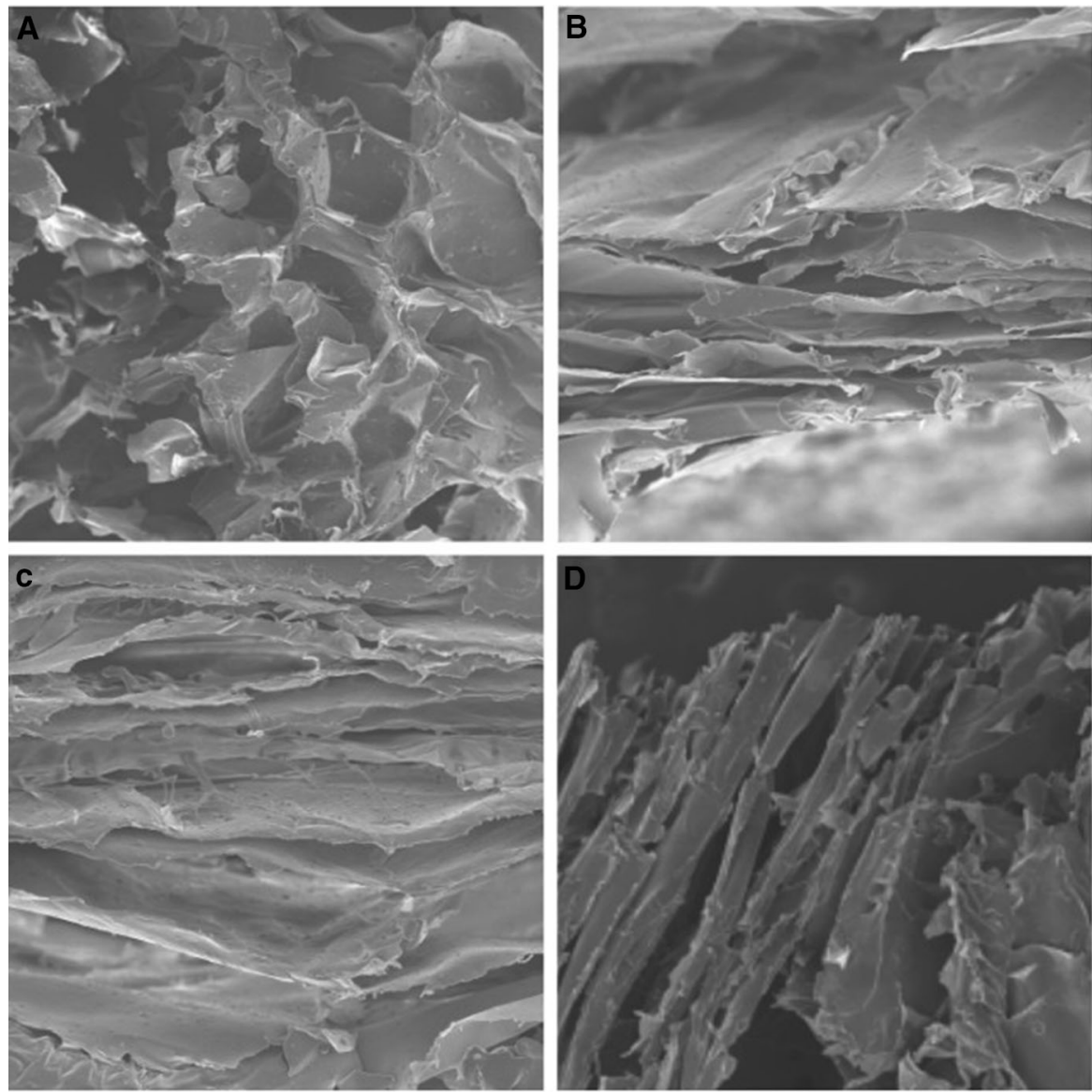

Fig. 2 Scanning electron micrographs of the cross-linked DNEM/chitosan scaffolds. a Scaffold cross-linked by GP; b scaffold crosslinked by GTA; c Scaffold cross-linked by UV; d scaffold not cross-linked

Crosslinking degree tests

The results showed that the cross-linking degree of the samples crosslinked by GP and GTA were $75.13 \pm 4.87 \%, 71.25 \pm 5.06 \%$, respectively. The differences between them were not statistically significant $(p \geq 0.05$ ). The cross-linking degree of the samples crosslinked by the UV and the sample not crosslinked were not statistically significant ( $p$ $\geq 0.05$ ), too (Fig. 3c). 

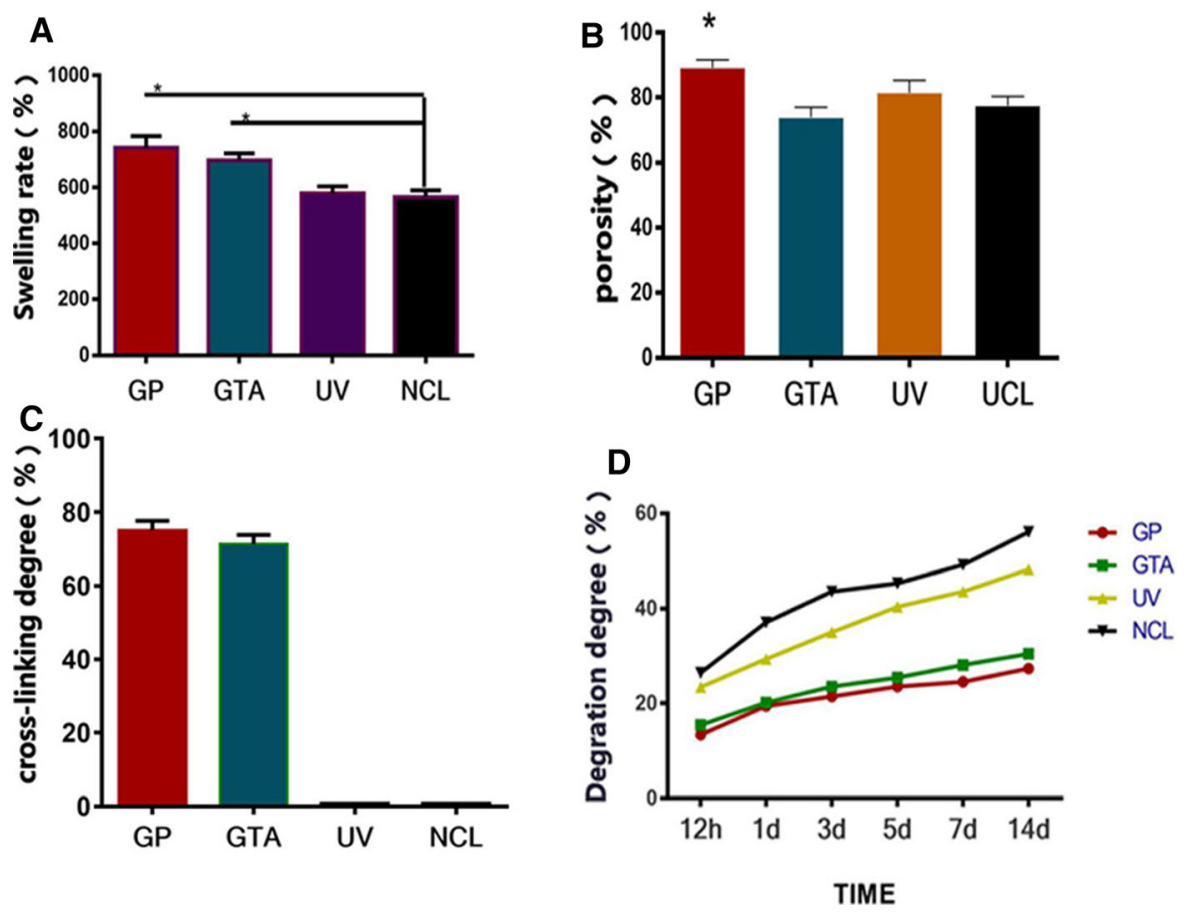

Fig. 3 Physicochemical property of the cross-linked DNEM/chitosan scaffolds. a Swelling degree of the scaffolds; $\mathbf{b}$ porosity rate of the scaffolds; c Crosslinking degree of the scaffolds; $\mathbf{d}$ dissolution degree of the scaffolds

Dissolution tests

The dissolution tests showed that dissolution behaviour of the sample crosslinked in different method varied. Diddolution degree of the samples crosslinked by GP, GA, UV and the sample not crosslinked increasing time, reaching a value of $27.36+1.49 \%$, $30.46 \%+2.15 \%, 48.23+1.16 \%$ and $56.12+2.2 \%$, respectively after $14 \mathrm{~d}$. The differences The degradation rates of the samples crosslinked by GP, GTA was lower than that of the samples crosslinked by UV and the sample not crosslinked. The differances were statistically significant $(p<0.05)$. The differences between the degradation rates of the samples crosslinked by GP, GTA were not statistically significant $(p \geq 0.05)$. The differences between the degradation rates of the samples crosslinked by UV and the sample not crosslinked were also not statistically significant $(p \geq 0.05)$ (Fig. 3d).

\section{Cytotoxicity and cytocompatibility tests}

For all groups, cells attached and distributed evenly on the scaffolds, number of cells adhered to the scaffolds crosslinked by GP were most (Fig. 4a). and a small amount of cells adhered to the scaffolds crosslinked by the GTA and UV (Fig. 4b, c), while there was the least cells on the sample not crosslinked (Fig. 4d).

Number of cells determined by CCK- 8 assay was a statistically significant difference between the different groups at each time point $(p<0.05)$. There were no statistical differences among absorbance value of GP crosslinked group, UV crosslinked group and negative control group at each time point. Absorbance values of not crosslinked group and GTA crosslinked group were significantly lower than that of GP crosslinked group, UV crosslinked group and negative control group.

Cytotoxic grading of GP crosslinked group, UV crosslinked group were 0 , cytotoxic grading of not crosslinked group was 1 , while cytotoxic grading of GTA crosslinked group was 2 (Fig. 4e).

Histocompatibility test

After the materials was implanted into the subcutaneous tissue of rats, the rats in each group had no abnormal eating, drinking and activities, there was no 

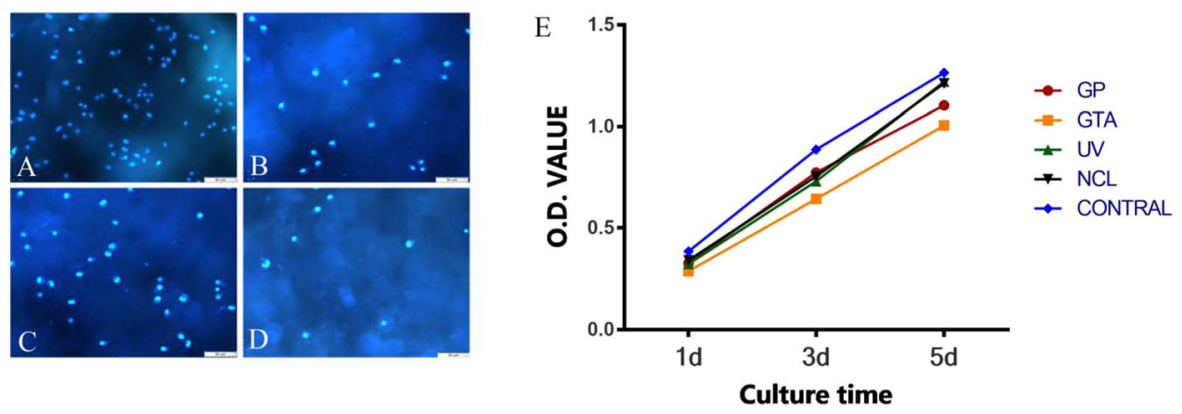

Fig. 4 Cytotoxicity and cytocompatibility of the cross-linked DNEM/chitosan scaffolds. a Cells adhered to the scaffolds crosslinked by GP; $\mathbf{b}$ cells adhered to the scaffolds crosslinked

swelling, sclerosis, leachate, and infection at the incision.

At 1 week after surgery, HE staining showed that a large number of inflammatory cells gathered around the scaffold material (Fig. 5b), which implied the inflammatory reaction of the GTA group was serious. And in the GP group, the UV group and the NCL group, a small amount of inflammatory cells were scattered around the scaffold (Fig. 5a, c, and d). At 4 week after surgery, the scoffolds of UV group and the NCL group had been completely degraded and absorbed, while the scaffold residue of GP and GTA group still observed. And the inflammatory reaction of GTA group decreased compared with that at $1 \mathrm{w}$, but still some inflammatory cells around the scaffolds (Fig. 5e). Almost no inflammatory cells were observed in GP group (Fig. 5f).

\section{Discussion}

Biomaterial scaffolds can give guidance cues to promote cell survival and axon growth through the injury field contains inhibitory molecular cues (Dietzmeyer et al. 2019; Johnson et al. 2013). Nerve or spinal cord extracellular matrix is used as materials to reduce these barriers and provide a multimodal approach to bridge the gap and promote tissue repair (Liu et al. 2013; Schnell et al. 2007). And ECM in the form of hydrogels is acceptable in clinical practice to fill the lesion with irregular and unpredictable geometry and offer minimally invasive delivery techniques.

In this study, we prepared a moldable nerve-repair scaffold by blending NECM and CS to fill the irregular cysts in SCI. To cover the shortage of scaffold with by GTA; c cells adhered to the scaffolds crosslinked by UV; d cells adhered to the scaffolds not crosslinked. e absorbance values of CCK-8 assay about cytotoxicity of the scaffolds

weak structural stability rapid degradation in vivo, the feasibility of three kinds of crosslinking motheds was evaluated to select the optimal way to enhance the stability in vivo.

Genipin, glutaraldehyde and UV-irradiation are commonly used for crosslinking of natural scaffolds. All of three crosslinking agents bind chitosan or proteins. Crosslinking degree and stability of the scaffold crosslinked by various crosslinking agents are different. Observed by SEM, the scaffold crosslinked by genipin showed a homogeneous porous structure, while the GA group and the UV crosslinked group showed multilayer sheet structure. The reason was that the scaffold material first formed a stable hydrogel under the action of genipin, and the gel occurred before the solid-liquid phase separation, so the porous sponge liked structure formated. The scaffolds of other three groups directly experience the process of solidliquid separation, the scaffolds is affected by the crystallization of glacial acetic acid, showing a flake or layer. Because of the high crosslinking degree of the GA group, compared with the UV and NCL group, the scaffolds showed a tighter and orderly arrangement between layers. The porosity rate of all the scaffolds of four groups produced in this study was more than $80 \%$. Cell proliferation and migration is known to be connected with surfaces and porosity of the material (Vila-Sanjurjo et al. 2019). The transportation of the metabolites and nutrients of the cells is improved with the increased of the porosity, which is beneficial to the migration and proliferation of the cells. But as the porosity increased, the strength of the scaffold will be weakened accordingly (Biswas et al. 2017; Kang et al. 2017). The porosity rate of the GP group was higher than that of the other three groups. The result also 

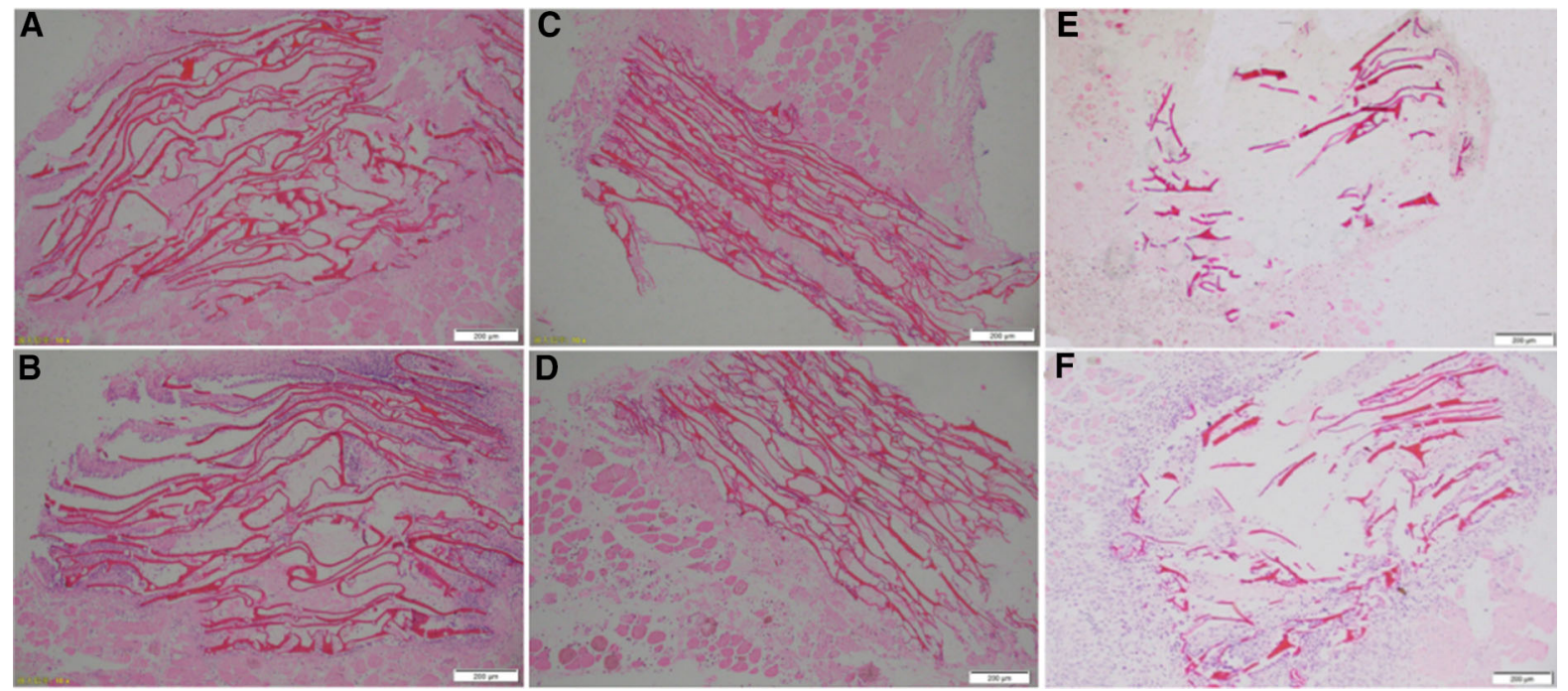

Fig. $5 \mathrm{H} \& \mathrm{E}$-stained histologic sections of the materials was implanted into the subcutaneous tissue of rats at various time. a Scaffold cross-linked by GP at 1 week after surgery; b scaffold cross-linked by GTA at 1 week after surgery; c scaffold cross-

proved the scaffold crosslinked by genipin was more beneficial to cells penetrate and adhere than the other groups.

Furthermore, the scaffold pore sizes also influence the function of cells for they play an essential role in nutrient and oxygen diffusion and waste removal. And the ideal pore sizes for cell adhesion, cell-cell interaction, cell transmigration and differentiation depending on the various tissue regeneration. It was shown that PCL scaffolds with a pore size of $125 \mu \mathrm{m}$ best supported PC12 cells neural differentiation in vitro (Vijayavenkataraman et al. 2018). The axon growth along the longitudinal direction of 3D hydrogel alginate displayed the highest axon density within the scaffold capillary diameter of 71-86 $\mu \mathrm{m}$ (Pawar et al. 2011). It was found that glial and axonal grew better on the pore sizes of collagen I scaffold ranging from 20 to $50 \mu \mathrm{m}$ (Bozkurt et al. 2009). And collagen membrane with pore sizes of $100 \mu \mathrm{m}$ was proper for the transplantation and differentiation of neural stem cells (Yuan et al. 2014). As a comprehensive analysis of previous studies, the ideal pore sizes of scaffold for nerve regeneration are depending on the origin of biomaterial. Scaffolds with pore sizes around $100 \mu \mathrm{m}$ seem to be more suitable for the regeneration of neurons (Bruzauskaite et al. 2016). In this study, the scaffold crosslinked by GP showed a uniform honeycomb porous structure with the pore sizes about linked by UV at 1 week after surgery; d scaffold not crosslinked at 1 week after surgery; e scaffold cross-linked by GP at 4 week after surgery; f scaffold cross-linked by GTA at 4 week after surgery $($ bar $=200 \mu \mathrm{m})$

$85.32+5.34 \mu \mathrm{m}$ which were closed to the reported ideal pore sizes. We will prepare the scaffold with different pore sizes and screen out the scaffold with best pore sizes for nerve regeneration in further study.

To evaluate the water absorption and retention of the scaffold, swelling ratio of the scaffolds were measured. The material with high swelling ratio has a better hydrophilic group and porous structure. After implantation, the scaffold with better liquid affinity can keep body fluid between the pores and provide the more necessary nutrients for cells (Hong et al. 2017; Huang et al. 2018). At the same time, the swelled scaffold provide greater space for cell adhesion and migration and a better local growth microenvironment (Wang et al. 2017). The swelling ratio of the scaffolds crosslinked by genipin and glutaraldehyde was $741+85.04 \%$ and $696.75+50.4 \%$ respectively, which was higher than that of UV group and the NCL group $(578.50+50.78 \%, 565.40+49.36)$. Then the scaffold crosslinked by genipin can provide a better microenvironment for cells.

The main aim of crosslinking is to obtain better mechanical strength and anti enzymatic ability. With the increase of crosslinking degree, mechanical strength will increase, while the tensile strength and elongation will decrease. The ideal scaffold should maintain a both elasticity and mechanical strength (Chen et al. 2009; Chiono et al. 2008; Li et al. 2015). 
Measured by ninhydrin method, the crosslinking ability of GP went near to GA, was $75.13+4.87 \%$ and $71.25+5.06 \%$, respectively. Dissolution tests showed that the scaffolds crosslinked by the GP group and the GA group degradated slowly relatively. The degradation rates of them were lower than that of the NCL group and the UV group. After implanted in the body, the scaffold can be degraded by the various components, especially the lysozyme and cathepsin. The dissolution tests reflected that scaffold crosslinked by the GP group and the GA group had better ability to resist the enzyme compared to the UV group and the NCL group. Therefore, their structure and morphology can be maintained and play the role in mediating cell and tissue growth and guiding the axon regeneration within a certain period.

As a material implanted in the body, the scaffold must meet the standard of cytotoxicity. The cytotoxicity test in vitro is a fast, standardized, and sensitive method to determine whether the material contains significant amounts of extractable substances that may harm the host. And the results showed that a strong correlation with those in vivo studies of short-term implants (Zhang et al. 2014, 2018). In this study, CCK-8 assay of the proliferation of L929 cells used to determine the cytotoxicity in vitro, which is recommended by the international standardization organization (ISO). There were no significant cytotoxicity of the scaffolds of the GP group and the UV group, while the proliferation of L929 cells was significant influenced by the scaffold of GA group. The cells grew well in the scaffold crosslinked by GP, while the scaffold of NCL group also had an effect on the cells. Maybe the scaffold of NCL group which was lack of stability, dissolve in the culture medium during the extraction. The intrinsic components of the medium were affected, and inhibited the proliferation of the cells.

When scaffolds were implanted in the muscles of rats, the scaffolds crosslinked by GP, UV-irradiation and the NCL showed good histocompatibility while there was inflammatory reaction around the scaffold crosslined by GA. And the scaffolds of GP group and GA group still remained at 4 weeks, while that of UV group and the NCL group have been degraded completely.

In summary, we used GP, GA and UV-irradiation to crosslink chitosan and NECM in this study. Both GP and GA enhanced scaffold stability in vitro and in vivo, while the effective crosslinking degree of UV is very low. What's more, the scaffold crosslinked by GP had a lower cytotoxicity and better biocompatibility than that of scaffold crosslinked by GA. To evaluate the feasibility of the NECM/CS crosslinked by GP in treatment of SCI repair, further in vitro and in vivo study is required.

\section{Conclusion}

The genipin crosslinked nerve acellular matrix/chitosan scaffold has more excellent microstructure, mechanical properties and biocompatibility, which is the best crosslinking scheme. Then GP was turning to be a safe and effective technology to crosslink nerve acellular matrix/chitosan scaffold.

Acknowledgements This work was supported by the following grants: National Natural Science Fund of China (Nos. 81870985, 31700930), Natural Science Foundation of Shandong Province (Nos. ZR2017MC052, BS2015SW021, ZR2016HP13, ZR2016HL18), the Project of Shandong Province Higher Educational Science and Technology Program (J18KA258), the Project of Science and Technology of Binzhou MedicalUniversity (Nos. BY2014KYQD25, BY2014KJ08).

\section{Compliance with ethical standards}

Conflict of interest The authors declare that they have no conflict of interest.

\section{References}

Badenhorst M, Verhagen E, Lambert M, van Mechelen W, Brown J (2019) When this happens, you want the best care: players' experiences of barriers and facilitators of the immediate management of rugby-related acute spinal cord injury. Qual Health Res 29:1862-1876

Barclay L, McDonald R, Lentin P, Bourke-Taylor H (2016) Facilitators and barriers to social and community participation following spinal cord injury. Aust Occup Ther J 63:19-28

Biswas P, Sen D, Mazumder S, Melo JS, Basak CB, Dasgupta K (2017) Porous nano-structured micro-granules from silicamilk bi-colloidal suspension: Synthesis and characterization. Colloids Surf B Biointerfaces 154:421-428

Bozkurt A, Deumens R, Beckmann C, Olde DL, Schugner F, Heschel I, Sellhaus B, Weis J, Jahnen-Dechent W, Brook GA et al (2009) In vitro cell alignment obtained with a Schwann cell enriched microstructured nerve guide with longitudinal guidance channels. Biomaterials 30:169-179 
Bruzauskaite I, Bironaite D, Bagdonas E, Bernotiene E (2016) Scaffolds and cells for tissue regeneration: different scaffold pore sizes-different cell effects. Cytotechnology 68:355-369

Chen MC, Liu CT, Tsai HW, Lai WY, Chang Y, Sung HW (2009) Mechanical properties, drug eluting characteristics and in vivo performance of a genipin-crosslinked chitosan polymeric stent. Biomaterials 30:5560-5571

Chiono V, Pulieri E, Vozzi G, Ciardelli G, Ahluwalia A, Giusti P (2008) Genipin-crosslinked chitosan/gelatin blends for biomedical applications. J Mater Sci Mater Med 19:889-898

Cripps RA, Lee BB, Wing P, Weerts E, Mackay J, Brown D (2011) A global map for traumatic spinal cord injury epidemiology: towards a living data repository for injury prevention. Spinal Cord 49:493-501

Dietzmeyer N, Forthmann M, Leonhard J, Helmecke O, Brandenberger C, Freier T, Haastert-Talini K (2019) Twochambered chitosan nerve guides with increased bendability support recovery of skilled forelimb reaching similar to autologous nerve grafts in the rat $10 \mathrm{~mm}$ median nerve injury and repair model. Front Cell Neurosci 13:149

Fajardo JE, Shrestha R, Gil N, Belsom A, Crivelli SN, Czaplewski C, Fidelis K, Grudinin S, Karasikov M, Karczynska AS et al (2019) Assessment of chemical-crosslinkassisted protein structure modeling in CASP13. Proteins 87:1283-1297

Fang Y, Zhang T, Song Y, Sun W (2019) Assessment of various crosslinking agents on collagen/chitosan scaffolds for myocardial tissue engineering. Biomed Mater 15(4):045003

Gao S, Yuan Z, Guo W, Chen M, Liu S, Xi T, Guo Q (2017) Comparison of glutaraldehyde and carbodiimides to crosslink tissue engineering scaffolds fabricated by decellularized porcine menisci. Mater Sci Eng C Mater Biol Appl 71:891-900

Hong L, Kim YM, Park HH, Hwang DH, Cui Y, Lee EM, Yahn S, Lee JK, Song SC, Kim BG (2017) An injectable hydrogel enhances tissue repair after spinal cord injury by promoting extracellular matrix remodeling. Nat Commun 8:533

Huang Q, Qin W, Garoushi S, He J, Lin Z, Liu F, Vallittu PK, Lassila L (2018) Physicochemical properties of discontinuous S2-glass fiber reinforced resin composite. Dent Mater J 37:95-103

Johnson PJ, Wood MD, Moore AM, Mackinnon SE (2013) Tissue engineered constructs for peripheral nerve surgery. Eur Surg 45(3):122-135

Kang X, Zhou M, Wang S, Jin S, Sun G, Zhu M, Jin R (2017) The tetrahedral structure and luminescence properties of Bi-metallic Pt1Ag28(SR)18(PPh3)4 nanocluster. Chem Sci 8:2581-2587

Kashif M, Jones S, Darain H, Iram H, Raqib A, Butt AA (2019) Factors influencing the community integration of patients following traumatic spinal cord injury: a systematic review. J Pak Med Assoc 69:1337-1343

Lau YT, Kwok LF, Tam KW, Chan YS, Shum DK, Shea GK (2018) Genipin-treated chitosan nanofibers as a novel scaffold for nerve guidance channel design. Colloids Surf B Biointerfaces 162:126-134
Leiva O, Leon C, Kah NS, Mangin P, Gachet C, Ravid K (2018) The role of extracellular matrix stiffness in megakaryocyte and platelet development and function. Am J Hematol 93:430-441

Lewandowska-Lancucka J, Gilarska A, Bula A, Horak W, Latkiewicz A, Nowakowska M (2019) Genipin crosslinked bioactive collagen/chitosan/hyaluronic acid injectable hydrogels structurally amended via covalent attachment of surface-modified silica particles. Int $\mathrm{J}$ Biol Macromol 136:1196-1208

Li Q, Wang X, Lou X, Yuan H, Tu H, Li B, Zhang Y (2015) Genipin-crosslinked electrospun chitosan nanofibers: determination of crosslinking conditions and evaluation of cytocompatibility. Carbohydr Polym 130:166-174

Lin T, Liu S, Chen S, Qiu S, Rao Z, Liu J, Zhu S, Yan L, Mao H, Zhu Q et al (2018) Hydrogel derived from porcine decellularized nerve tissue as a promising biomaterial for repairing peripheral nerve defects. Acta Biomater 73:326-338

Liu Y, Kim HI (2012) Characterization and antibacterial properties of genipin-crosslinked chitosan/poly(ethylene glycol)/ZnO/Ag nanocomposites. Carbohydr Polym 89:111-116

Liu J, Chen J, Liu B, Yang C, Xie D, Zheng X, Xu S, Chen T, Wang L, Zhang Z et al (2013) Acellular spinal cord scaffold seeded with mesenchymal stem cells promotes longdistance axon regeneration and functional recovery in spinal cord injured rats. J Neurol Sci 325:127-136

Meyer C, Stenberg L, Gonzalez-Perez F, Wrobel S, Ronchi G, Udina E, Suganuma S, Geuna S, Navarro X, Dahlin LB et al (2016) Chitosan-film enhanced chitosan nerve guides for long-distance regeneration of peripheral nerves. Biomaterials 76:33-51

Milbreta U, von Boxberg Y, Mailly P, Nothias F, Soares S (2014) Astrocytic and vascular remodeling in the injured adult rat spinal cord after chondroitinase ABC treatment. J Neurotrauma 31:803-818

Pawar K, Mueller R, Caioni M, Prang P, Bogdahn U, Kunz W, Weidner N (2011) Increasing capillary diameter and the incorporation of gelatin enhance axon outgrowth in alginate-based anisotropic hydrogels. Acta Biomater 7:2826-2834

Saldin LT, Cramer MC, Velankar SS, White LJ, Badylak SF (2017) Extracellular matrix hydrogels from decellularized tissues: Structure and function. Acta Biomater 49:1-15

Schnell E, Klinkhammer K, Balzer S, Brook G, Klee D, Dalton P, Mey J (2007) Guidance of glial cell migration and axonal growth on electrospun nanofibers of poly-epsiloncaprolactone and a collagen/poly-epsilon-caprolactone blend. Biomaterials 28:3012-3025

Starnecker F, Konig F, Hagl C, Thierfelder N (2018) Tissueengineering acellular scaffolds-The significant influence of physical and procedural decellularization factors. J Biomed Mater Res B Appl Biomater 106:153-162

Tian T, Yu Z, Zhang N, Chang Y, Zhang Y, Zhang L, Zhou S, Zhang C, Feng G, Huang F (2017) Modified acellular nerve-delivering PMSCs improve functional recovery in rats after complete spinal cord transection. Biomater Sci 5:2480-2492

Tukmachev D, Forostyak S, Koci Z, Zaviskova K, Vackova I, Vyborny K, Sandvig I, Sandvig A, Medberry CJ, Badylak 
SF et al (2016) Injectable extracellular matrix hydrogels as scaffolds for spinal cord injury repair. Tissue Eng Part A 22:306-317

Vijayavenkataraman S, Zhang S, Thaharah S, Sriram G, Lu WF, Fuh JYH (2018) Electrohydrodynamic jet 3D printed nerve guide conduits (NGCs) for peripheral nerve injury repair. Polymers 10(7):753

Vila-Sanjurjo C, David L, Remunan-Lopez C, Vila-Sanjurjo A, Goycoolea FM (2019) Effect of the ultrastructure of chitosan nanoparticles in colloidal stability, quorum quenching and antibacterial activities. J Colloid Interface Sci 556:592-605

Vyborny K, Vallova J, Koci Z, Kekulova K, Jirakova K, Jendelova P, Hodan J, Kubinova S (2019) Genipin and EDC crosslinking of extracellular matrix hydrogel derived from human umbilical cord for neural tissue repair. Sci Rep 9:10674

Wang Q, Zhang C, Zhang L, Guo W, Feng G, Zhou S, Zhang Y, Tian T, Li Z, Huang F (2014) The preparation and comparison of decellularized nerve scaffold of tissue engineering. J Biomed Mater Res A 102:4301-4308

Wang S, Guan S, Zhu Z, Li W, Liu T, Ma X (2017) Hyaluronic acid doped-poly(3,4-ethylenedioxythiophene)/chitosan/ gelatin (PEDOT-HA/Cs/Gel) porous conductive scaffold for nerve regeneration. Mater Sci Eng C Mater Biol Appl 71:308-316

Yuan N, Tian W, Sun L, Yuan R, Tao J, Chen D (2014) Neural stem cell transplantation in a double-layer collagen membrane with unequal pore sizes for spinal cord injury repair. Neural Regen Res 9:1014-1019

Zhang N, Zhou M, Zhang Y, Wang X, Ma S, Dong L, Yang T, Ma L, Li B (2014) Porcine bone grafts defatted by lipase: efficacy of defatting and assessment of cytocompatibility. Cell Tissue Bank 15:357-367

Zhang N, Ma L, Liu X, Jiang X, Yu Z, Zhao D, Zhang L, Zhang C, Huang F (2018) In vitro and in vivo evaluation of xenogeneic bone putty with the carrier of hydrogel derived from demineralized bone matrix. Cell Tissue Bank 19:591-601

Zhou X, Cai Q, Yan N, Deng X, Yang X (2010) In vitro hydrolytic and enzymatic degradation of nestlike-patterned electrospun poly(D, L-lactide-co-glycolide) scaffolds. J Biomed Mater Res A 95:755-765

Publisher's Note Springer Nature remains neutral with regard to jurisdictional claims in published maps and institutional affiliations. 